AperTO - Archivio Istituzionale Open Access dell'Università di Torino

\title{
Glass-Ceramic Scaffolds and Shock Waves Effect on Cells Migration
}

\section{This is the author's manuscript}

Original Citation:

Availability:

This version is available http://hdl.handle.net/2318/108468

since

Published version:

DOI:10.4028/www.scientific.net/KEM.361-363.233

Terms of use:

Open Access

Anyone can freely access the full text of works made available as "Open Access". Works made available under a Creative Commons license can be used according to the terms and conditions of said license. Use of all other works requires consent of the right holder (author or publisher) if not exempted from copyright protection by the applicable law. 


\title{
Glass-ceramic Scaffolds and Shock Waves Effect on Cells Migration
}

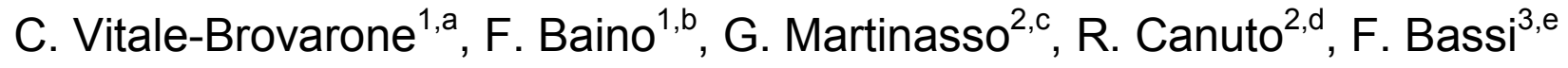 \\ and $\mathrm{E}$. Verné ${ }^{1, \mathrm{f}}$ \\ ${ }^{1}$ Politecnico di Torino, Materials Science and Chemical Engineering Department, Corso Duca degli \\ Abruzzi 24, 10128 Torino, Italy \\ ${ }^{2}$ Department of Experimental Medicine and Oncology, University of Turin, Italy \\ ${ }^{3}$ Department of Human Oncology and Biomedical Sciences, School of Dentistry, University of \\ Turin, Italy \\ achiara.vitale@polito.it, bfrancesco.baino@polito.it, 'cgermana.martinasso@unito.it, \\ drosangela.canuto@unito.it, ${ }^{\mathrm{d}}$ francesco.bassi@unito.it, ${ }^{\mathrm{f}}$ enrica.verne@polito.it
}

Keywords: glass-ceramic scaffolds, bone grafting, osteoblasts migration, shock -waves

\begin{abstract}
Glass-ceramic scaffolds for bone grafting have been prepared using the sponge replication method and a highly bioactive silica based glass belonging to the system $\mathrm{SiO}_{2}-\mathrm{P}_{2} \mathrm{O}_{5}$ $\mathrm{CaO}-\mathrm{MgO}-\mathrm{Na}_{2} \mathrm{O}-\mathrm{K}_{2} \mathrm{O}$ (CEL2). The parameters for the sponge impregnation were optimized in order to obtain a continuous coating of the polymeric skeleton which will lead, after the polymeric phase removal, to a resistant macroporous glass-ceramic template. A complete characterization was carried out on the obtained scaffold in order to assess its morphology and specifically its porosity and the degree of pores interconnection. A human osteoblasts cells line (MG-63) was cultured onto the scaffold and the effect of stimulation with shock waves on the cell ability of colonizing the scaffold was investigated.
\end{abstract}

\section{Introduction}

Bone substitutes are frequently needed in many orthopaedic, maxillofacial and craniofacial surgeries due to trauma and tumors. The physiological regeneration of bone is not sufficient if the bone defect exceeds the critical size and thus a bone graft is needed. Limitation and drawbacks of autografts, allografts and xenografts are known and widely reported in literature [1]. An optimal scaffold for bone tissue engineering should fulfill the following criteria: be osteoconductive or even better osteoproductive and acts as a template to provide structural support to the newly formed bone. For this purpose, open pores at least $100 \mu \mathrm{m}$ wide highly interconnected to allow cell migration, tissue in-growth and vascularisation are needed. An optimal scaffold should also provide sufficient mechanical strength matching that of the host bone and should bond to it without the formation of scar tissue, generating a stable interface. Among bioceramics hydroxyapatite, $\beta$-tricalcium phosphate and combinations of them have been studied as scaffolding materials due to their chemical analogy with the inorganic component of bone. More recently, glasses or glass-ceramics have been investigated as candidates for bone grafts. In fact glasses can be Class A bioactive materials which mean they are able to bond to both hard and soft tissues [2]. Bioactive glassceramic scaffolds with a high amount of pores within $100-500 \mu \mathrm{m}$ highly interconnected and in amount higher than $60-70 \%$ vol. can be prepared through the sponge impregnation method [3]. These scaffolds are interesting and valid candidates for stimulating the bone regeneration and for supporting the newly formed bone. In the present research work glass-ceramic scaffolds were successfully obtained through the sponge impregnation method and were tested in vitro to assess their biocompatibility. In particular, cells exposed to shock waves (SWs) were seeded onto the scaffolds in order to verify the effect of SWs on cells migration within the scaffold and on bone production. For comparative purposes, also not treated cells were seeded onto the glass-ceramic scaffolds. 


\section{Materials and Methods}

A glass belonging to the system $\mathrm{SiO}_{2}-\mathrm{P}_{2} \mathrm{O}_{5}-\mathrm{CaO}-\mathrm{MgO}-\mathrm{Na}_{2} \mathrm{O}-\mathrm{K}_{2} \mathrm{O}$ was prepared through traditional melting and quenching technique. Glass powders were mixed with water and poly-vinyl alcohol obtaining a suspension that was used to impregnate a polyurethane sponge that was subsequently thermally treated to remove the organic part and to sinter the inorganic one. The obtained 3Dscaffolds were characterised through SEM, EDS and XRD before and after soaking in Simulated Body Fluid (SBF). The porosity was investigated through image analysis on different scaffold cross sections. The scaffolds $\left(1 \times 1 \times 1 \mathrm{~cm}^{3}\right)$ were soaked in SBF for 1 week before cell seeding to test their bioactivity and to stimulate the precipitation of an hydroxyapatite layer, known to favour bone formation. Specifically, MG-63 $(90000 / \mathrm{ml})$ cells were exposed to SWs at different energy levels ranging from 0.08 to $0.32 \mathrm{~mJ} / \mathrm{mm}^{2}$; the SWs generator utilized was a piezoelectric device (Piezoson 100, Richard Wolf, Knittlingen, Germany) designed for clinical use in orthopaedics and traumatology. For each energy level, different numbers of impulses were tested (from 50 to 1000 at 4 impulses/s). After these preliminary experiments aimed to individualize the SWs exposure able to increase cell proliferation, only SWs treatment corresponding to $0.22 \mathrm{~mJ} / \mathrm{mm}^{2}$ and 100 total impulses was used. The sterilized scaffolds $\left(1 \mathrm{~cm}^{3}\right)$, pre-treated in SBF for 1 week, were preconditioned for 24 hours in multiwells $\left(9 \mathrm{~cm}^{2}\right.$ area) containing culture medium used to grow MG-63 cells. After removing preconditioning medium, cells, treated or not with SWs, were seeded $\left(10000\right.$ cells $\left./ \mathrm{cm}^{2}\right)$ on the scaffolds. 6,10 and 20 days after osteoblast seeding, the medium was removed and the scaffolds were used to count the cells grown inside, to analyze their morphology and to evaluate the presence of calcium nodules. Cells migration within the scaffold was assessed by observing different cross sections of the scaffold at SEM.

\section{Results and Discussion}

CEL2 glass-ceramic macroporous scaffolds were successfully obtained using the replication method. As can be observed in fig. 1 the obtained scaffolds have a 3D-porous structure similar to the trabecular bone structure with open pores ranging from $100 \mu \mathrm{m}$ up to $300 \mu \mathrm{m}$, highly interconnected. The thickness of the scaffold trabeculae is a few tenths of microns.

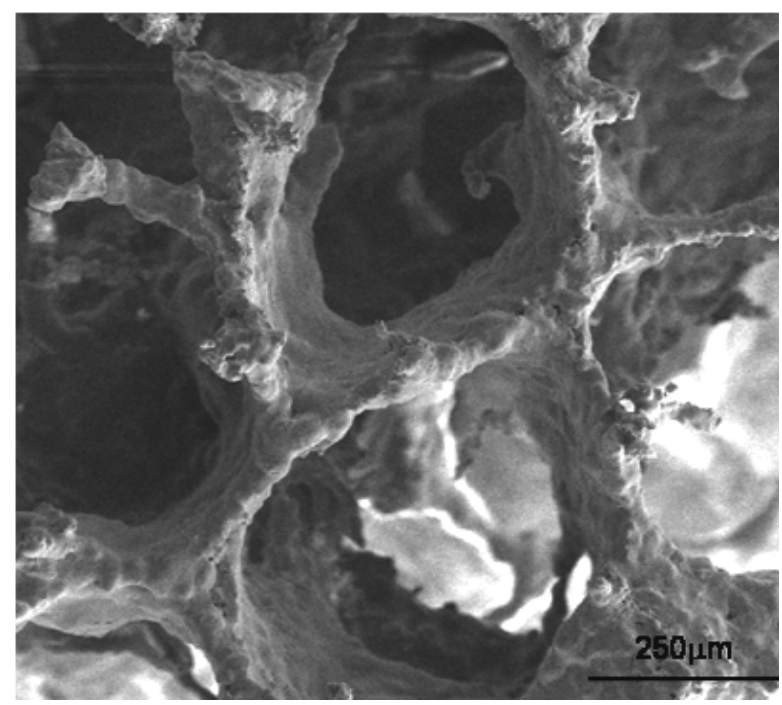

Fig. 1 3D-glass ceramic scaffold

The pores struts are well sintered as can be seen in fig. 2 and along the scaffold trabeculae a dispersed microporosity was observed due to the polymeric skeleton removal. The presence of a diffuse microporosity as well as the surface roughness of the scaffold due to its glass-ceramic nature are interesting features as they are known to be positive for protein and cell adhesion. 


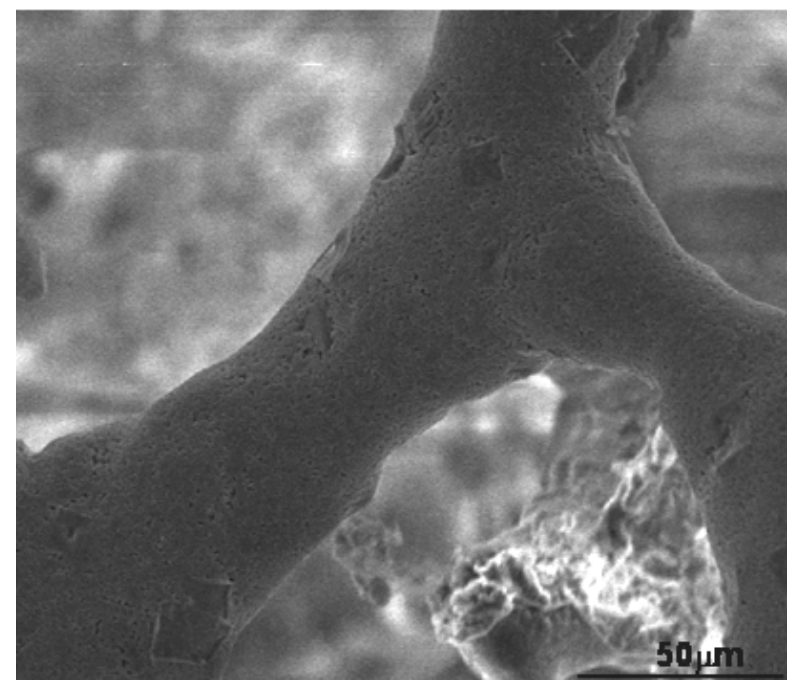

Fig. 2 Magnification of a scaffold trabecula

The total porosity was calculated through weight measurements on five different specimens showing values within $65-70 \%$ vol. and an average compression strength of $2 \mathrm{MPa}$ which are considered optimal for a bone substitute.

CEL2 scaffolds showed to be highly bioactive as their surface was completely covered by agglomerates of microcrystalline HAp after one week of soaking in SBF. At this purpose, fig. 3 reports a micrograph of a scaffold surface after 1 week in SBF colonized with not treated osteoblasts for 20 days. As can be observed, many HAp agglomerates are present on the scaffold surface assessing its bioactivity; cells attach and spread onto these HAp microcrystals.

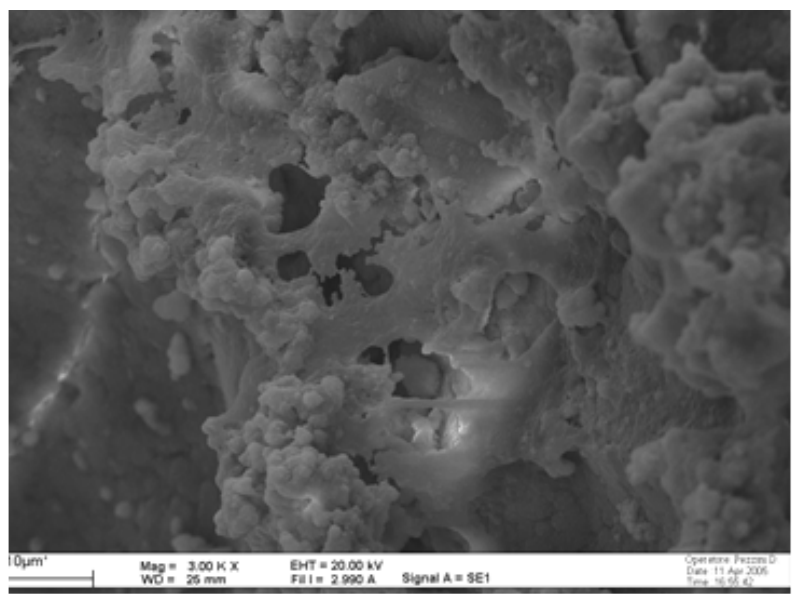

Fig. 3 Scaffold soaked in SBF for 1 week and colonized for 20 days with untreated cells

The osteoblasts, colonising the scaffold, strongly adhered to its porous structure and appeared to be attached with a close contact with the surface, adopting an extended morphology with numerous filopodia and lamellipodia. The osteoblasts spreading and migration within the scaffolds was evaluated by SEM analysis. Different cross sections of the scaffolds were obtained (up to $5 \mathrm{~mm}$ from the surface) to compare the depth of colonization of SWs-treated cells in comparison with untreated ones. SEM analysis showed that at 10 and 20 days SWs-treated cells were present in the deepest layers up to $5 \mathrm{~mm}$, whereas the control cells were present only at the surface. At this purpose, fig. 4 reports a micrograph of a scaffold cross-section at $3 \mathrm{~mm}$ from the outer surface after 10 days of culture with cells treated with SWs. 


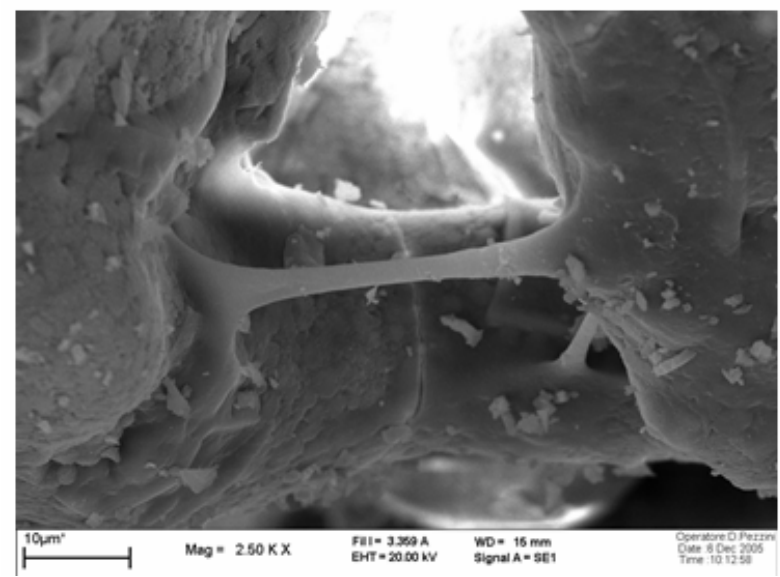

Fig. 4 Scaffold cross section after 10 days of culture with cells treated with SWs

Alizarin red staining for scaffolds seeded with SWs treated cells showed the presence of calcium nodules larger and in higher number than scaffold seeded with control cells. The rationale of having used SWs to induce bone synthesis derived also from other observations, and from increasingly application in various musculoskeletal disorders, even if the molecular mechanisms underlying SWs effectiveness remain rather unknown. In vitro studies on human osteoblasts-like cells evidenced that treatment with SWs influences cells proliferation enhancing the transmembrane currents, as well as the voltage dependence of Ca-activated and K channels. Animal studies suggested that SWs first stimulate the expression of several factors, including eNOS, VEGF and PCNA, then induce neovascularization improving blood supply and increasing cells proliferation $[4,5]$.

\section{Conclusions}

3D bioactive glass-ceramic scaffolds were successfully obtained through the sponge impregnation method using a polymeric template. Pores in the $100-500 \mu \mathrm{m}$ range were obtained in amount greater than $65 \%$ vol. with trabecular morphology analogous to spongious bone. The scaffolds are highly bioactive and possess satisfactory mechanical strength. Moreover, they are highly biocompatible and the stimulation of cells with shock waves has been shown to be effective in enhancing cells migration within the scaffold structure and calcium nodules formation.

\section{References}

[1] J.C. Banwart, M.A. Asher and R.S. Hassanein: Spine 20 (1995), p. 1055

[2] J. Jones and L.L. Hench: Curr. Opin. Solid State Mater. Sci. 7 (2003), p. 301

[3] C. Vitale-Brovarone, E. Verné, L. Robiglio, P. Appendino, F. Bassi, G. Martinasso, G. Muzio and R. Canuto: Acta Biomat. 3 (2007), p. 199

[4] L. Martini, G. Giavaresi, M. Fini, P. Torricelli, V. Borsari, R. Giardino, M. De Pretto, D. Remondini and G.C. Castellani: Int. J. Artif. Organs 28 (2005), p. 841

[5] C.G. Wang, F.S. Wang, K.D. Yang, L.H. Weng, Y.C. Sun and Y.J. Jang: Bone 32 (2003), p. 387 